\title{
Monseigneur Charles Larocque, évêque de Saint-Hyacinthe, et la Confédération
}

\section{Lucien Lemieux}

Volume 34, 1967

URI : https://id.erudit.org/iderudit/1007421ar

DOI : https://doi.org/10.7202/1007421ar

Aller au sommaire du numéro

Éditeur(s)

Les Éditions Historia Ecclesiæ Catholicæ Canadensis Inc.

ISSN

0318-6172 (imprimé)

1927-7067 (numérique)

Découvrir la revue

Citer cet article

Lemieux, L. (1967). Monseigneur Charles Larocque, évêque de Saint-Hyacinthe, et la Confédération. Sessions d'étude - Société canadienne d'histoire de l'Église catholique, 34, 55-61. https://doi.org/10.7202/1007421ar

Tous droits réservés @ Les Éditions Historia Ecclesiæ Catholicæ Canadensis Inc., 1968
Ce document est protégé par la loi sur le droit d'auteur. L'utilisation des services d'Érudit (y compris la reproduction) est assujettie à sa politique d'utilisation que vous pouvez consulter en ligne.

https://apropos.erudit.org/fr/usagers/politique-dutilisation/ 


\section{Monseigneur Charles Larocque, évêque de Saint-Hyacinthe, et la Confédération}

\section{INTRODUCTION}

Monseigneur Charles Larocque, né à Chambly le 15 novembre 1809 , fit ses études classiques et théologiques à Saint-Hyacinthe, puis à Montréal. Après avoir été vicaire et curé à différents endroits, il fut chargé en 1844 de la cure de Saint-Jean d'Iberville. C'est le 20 mars 1866 que le curé Larocque fut appelé à remplacer son cousin malade, Mgr Joseph Larocque, sur le siège épiscopal de Saint-Hyacinthe. Le nouvel évêque fut sacré dans l'église de Saint-Jean le 29 juillet suivant, et deux jours plus tard, il prit possession de sa cathédrale.

Des occasions se présentèrent qui amenèrent le nouvel évêque à s'adresser à des représentants du gouvernement, spécialement au procureur général Georges-Etienne Cartier; celui-ci avait d'ailleurs assisté à son sacre en l'église de Saint-Jean et était considéré par l'évêque comme son " honorable et bien digne $a^{2}{ }^{1}{ }^{1}$. Dès septembre 1866 , Mgr Larocque écrivit à Cartier au sujet de l'érection de la paroisse Sainte-Angèle ${ }^{2}$. Au mois d'avril suivant, après le retour de Cartier d'Angleterre où avait lieu la dernière conférence préparatoire à l'Acte confédératif, celui-ci avait reçu une autre lettre de l'évêque de SaintHyacinthe. Il y était question de la division de la paroisse de SaintMathias et de la nomination d'un nouveau régistrateur pour le comté d'Iberville. Puis l'évêque ajoutait à la fin de sa lettre:

Me serait-il permis de terminer par un mot de félicitations sur vos succès et vos triomphes en Angleterre? En cas que ce ne soit pas le lieu de le faire, je me contenterai de vous dire que j'en ai le désir et la volonté 3 .

\section{I. - D'accord avec la suggestion de Mgr Laflèche}

Une fois que l'on eut appris au Canada que l'Acte de l'Amérique du Nord britannique avait été sanctionné par la reine Victoria, l'évêque coadjuteur de Trois-Rivières, Mgr Laflèche, suggéra sous forme de question à l'évêque administrateur du siège archiépiscopal de Québec, Mgr Baillargeon:

Ne serait-il pas désirable que les évêques, ceux de la province de Québec au moins, publiassent, après la proclamation de l'Acte de la Confédération et avant les élections générales, un Mandement, dans leurs diocèses respectifs, pour engager tous les catholiques à accepter de bon cour le nouvel état de choses, à contribuer franchement à le

1 A.E.S.H., $R L$, Série I, 5, 137-138, 2 mars 1867, Mgr Larocque à l'honorable N. F. Belleau.

2 Ibid., 29-30, 6 sept.

3 Ibid., 171-173, 22 avril 1867. 
faire bien fonctionner, à se tenir étroitement unis, enfin à choisir soigneusement de bons membres ${ }^{4}$ ?

Il est intéressant de remarquer que cette proposition de Mgr Laflèche acceptée par Mgr Baillargeon, fut d'abord transmise à Mgr Langevin de Rimouski. C'est ce dernier, sacré évêque quelques semaines plus tôt, c'est-à-dire au début de mai, qui fit connaître à Mgr Larocque la suggestion de Mgr Laflèche et qui l'incita à obtenir l'assentiment de Mgr Bourget de Montréal là-dessus ${ }^{5}$.

L'évêque de Saint-Hyacinthe rencontra celui de Montréal quelques jours plus tard. Mgr Bourget était déjà en train de faire imprimer une lettre circulaire pour son clergé; il y était uniquement question de la conduite à tenir lors des élections générales qui suivraient la mise en vigueur de l'Acte confédératif. Selon Mgr Larocque, l'évêque de Montréal n'écrivait cependant pas un mot " en faveur du nouvel ordre des choses, le mot de Confédération ne se trouvant même pas dans la lettre $^{6}$ ». En fait, Mgr Bourget avait tout de même énoncé le principe suivant: " tous les sujets étaient obligés en conscience de se soumettre à tout gouvernement légitimement établi ${ }^{7}$." $\mathrm{Ce}$ ne semblait pas suffisant aux yeux de Mgr Larocque.

Pour sa part, celui-ci répondit à l'évêque de Rimouski qu'il se modèlerait sur Mgr Baillargeon de Québec à propos de ce "grand événement politique du jour, la Confédération 8 ". Il se mit d'ailleurs tout de suite à la tâche. Ainsi, le 13 juin, il écrivait à l'abbé Cazeau, vicaire général de Québec:

Je dois reprendre lundi prochain le travail de la visite pastorale; et avant de partir, je me suis occupé de faire un Mandement sur l'affaire de la Confédération, pour me conformer à ce qui avait été agréé entre Mgr de Tloa (Mgr Baillargeon) et quelques autres évêques de la province, selon que m'en a écrit Mgr Langevin. Je laisse entre les mains de mes trois grands vicaires le manuscrit préparé pour la circonstance, mais avec l'instruction de ne le faire imprimer, qu'en autant que mes idées ne feraient point un trop gros contraste avec celles de Mgr de Tloa. Et sur ce, l'on m'a demandé de tâcher de savoir au plus tôt comment votre illustre évêque aborderait la question, et avec quels détails il la traiterait...

J'ai lu avec attention le Mandement de l'évêque de Trois-Rivières, qui me paraît vraiment un document bien digne et bien fait. Ferezvous à Québec quelque chose du même genre? Il se trouve que mon travail s'accorde pour le fond avec ce Mandement 9 .

De fait, cinq jours plus tard, on publia la Lettre pastorale de $\mathrm{Mgr}$ Larocque concernant l'inauguration du Gouvernement fédéral.

4 A.E.S.H., II, C-2, 1867-1869, 25 mai 1867, Mgr J. Langevin à Mgr Larocque.

5 Ibid.

6 A.E.S.H., $R L$, Série I, 5, 203-205, 4 juin 1867, à Mgr Langevin.

7 M.E.M., V, 214.

8 A.E.S.H., RL, Série I, 5, 204, 4 juin 1867.

$9 \quad$ Ibid., 205, 13 juin 1867. 


\section{II. - Lettre Pastorale du 18 Juin}

Tout en ne voulant pas brimer la liberté d'opinion de ses fidèles, l'évêque de Saint-Hyacinthe prit fermement position en faveur du pacte fédéral, qui était d'ailleurs un état de fait et une loi dont la mise en vigueur aurait lieu le $\mathbf{1}^{\text {er }}$ juillet suivant. Il avoua que, par sa Lettre, il apparaîtrait peut-être plus comme un patriote que comme un évêque ${ }^{10}$, mais il croyait être de son devoir pastoral d'instruire et de diriger ses fidèles " dans tout ce qui tient à l'ordre religieux, parce que la base des sociétés chrétiennes, c'est la religion elle-même ${ }^{11}$ 》.

On peut retracer trois points principaux dans le document épiscopal.

Premièrement, Mgr Larocque invita ses fidèles à se mettre en garde contre les tendances de certains esprits et de certains journaux, parmi lesquels il incluait certainement, quoiqu'il n'en donnât pas le nom, le Courrier de Saint-Hyacinthe. Ce dernier était en effet décrit à peu près à la même date par l'évêque, dans une lettre adressée au cardinal préfet de la Propagande à Rome, comme un malheureux écho d'un journal de Montréal, Le Pays, tous deux \& défenseurs et propagateurs des doctrines ultralibérales de notre époque, qui sentent si fortement l'irréligion et l'impiété ${ }^{12}$ ». Mgr Larocque nomma cependant la société secrète, désignée sous le nom de Club de Saint-Jean-Baptiste, dont l'optique politique ressemblait à celle des journaux mentionnés. Leurs tendances s'exprimaient dans le slogan suivant: " Plutôt l'annexion que la confédération telle qu'elle nous est donnée. " Pour l'évêque, l'annexion aux Etats-Unis équivalait à la mort de la nation canadiennefrançaise.

Monseigneur Larocque n'ignorait pas cependant qu'il y avait des hommes pleins de bonne foi et de loyauté qui redoutaient la Confédération. C'est pourquoi il admettait en second lieu que la position du peuple canadien-français était un peu compliquée à cause de sa croyance et de ses mours. Mais au lieu d'être pour lui une occasion de défaitisme, c'était plutôt un stimulant: "Une occasion de lutte rencontrée avec courage et énergie, écrivait-il, est un moyen assuré de multiplier ses forces et de se faire respecter." De plus, il comptait sur la force de caractère de plusieurs personnalités canadiennes-françaises et sur l'influence tonifiante de certains journaux. En somme, Mgr Larocque demeurait optimiste, ce qui lui faisait affirmer que la Confédération ne lui apparaissait nullement comme un danger à redouter ${ }^{13}$.

$\mathrm{Au}$ fond, les inconvénients éventuels de la Confédération, l'évêque les prévoyait. Il craignait lui aussi de voir entamés les droits et les privilèges des Canadiens français. Mais cela ne dépendrait pas de la Confédération elle-même, et c'est son troisième point, mais du défaut

10 M.E.S.H., II, 18 juin 1867, 436.

11 Ibid., 422.

12 A.E.S.H., $R L$, Série I, 5, 197-200, au cardinal Barnabo.

13 M.E.S.H., II, 18 juin 1867, 424-429. 
principal de ses compatriotes, c'est-à-dire celui de se jalouser les uns les autres.

Et cette jalousie va jusqu'à nous porter à voir avec une espèce de peine et de regret, quelqu'un d'entre nous s'élever au-dessus des autres par un talent ou un mérite qu'on est cependant forcé de reconnaître.

Monseigneur Larocque invita donc ses fidèles à éviter la division et l'esprit de parti; il avait alors en vue les élections prochaines. Le remède à tous les abus serait une bienveillance mutuelle, faite d'union, de courage, d'énergie et de persévérance.

La Lettre pastorale se terminait par un panégyrique de l'acte confédératif, ce qui était en même temps une manière d'inciter les gens à voter pour les candidats qui y étaient favorables, en l'occurrence les conservateurs dont le chef de file au Québec était Georges-Etienne Cartier.

La confédération, mieux encore que l'union qu'elle remplace, servira nos intérêts et le développement de toutes nos ressources pour répandre partout la prospérité matérielle, sans toucher aucunement à nos intérêts religieux, ni à aucune de ces belles institutions qui sont véritablement l'honneur et le soutien de notre foi et de notre pays, et dont le soin et la conservation ne dépendent que de nousmêmes.

C'est pourquoi dans chaque paroisse, on devait célébrer une grand-messe précédée du Veni Creator Spiritus, un des dimanches suivants, en l'honneur de la nouvelle constitution gouvernementale ${ }^{14}$.

Vers la fin du mois d'août, des citoyens de Saint-Hyacinthe posèrent deux questions à l'évêque afin d'avoir plus de précisions.

Un électeur peut-il au point de vue de la conscience voter pour les candidats de l'opposition dans le cas où il croirait sincèrement que ce sont eux qui sont les plus capables de promouvoir les intérêts du pays?

Un électeur qui voterait pour un candidat de l'opposition devrait-il pour ce fait seul être privé des sacrements et de la sépulture ecclésiastique ${ }^{15}$ ?

Monseigneur Larocque leur répondit ne pas vouloir entrer dans les détails ni faire de la casuistique, d'autant plus que l'on était en plein temps d'élections; la votation avait en effet lieu le 23 août et le 18 septembre ${ }^{16}$. "La prudence me commande de ne rien ajouter à ma Lettre pastorale ", déclara l'évêque.

Celle-ci me paraît d'ailleurs renfermer un exposé de principes généraux parfaitement suffisant pour que les électeurs qui voudront l'étudier et en tenir compte, arrivent aisément à se former une conscience éclairée sur le vote qu'ils vont être appelés à donner dans le moment si grave et si solennel de leur prochaine apparition sur la plate-forme électorale ${ }^{17}$.

14 Ibid., 430-437.

15 A.E.S.H., XVII, C. 37, 1867, 26 août 1867, A. Malhiot, G. C. Dessaulles, J. B. Bourgeois à Mgr Larocque.

16 Jean Hamelin, John Huot, Marcel Hamelin, Aperçu de la politique canadienne au XIX ${ }^{e}$ siècle, Québec, Presses de l'Université Laval, 1965, 48.

17 A.E.S.H., $R L$, Série I, 5, 219-221, 27 août 1867. 
En réalité, la situation de l'évêque était délicate. Il n'est pas sûr que sa Lettre pastorale produisît tous les effets prévus. Si l'on jette un coup d'œil sur le résultat des élections dans le Québec, on constate qu'au moins quatre des seize députés libéraux élus étaient de son diocèse, c'est-à-dire les députés d'Iberville, de Rouville, de Shefford et de Saint-Hyacinthe ${ }^{18}$.

\section{III. - Intervention Infructueuse AUPRÈs DE Mgr Bourget}

Un mois environ avant les élections, Mgr Bourget de Montréal avait publié une Lettre pastorale. Il y exprimait à peu près dans les mêmes termes les idées déjà émises à son clergé à propos des élections. Il rappelait que l'Eglise catholique du Canada avait toujours accepté les divers gouvernements civils sous lesquels elle s'était trouvée et mentionna qu'encore aujourd'hui « elle acceptait sans réplique le gouvernement fédéral, parce qu'il émanait de la même autorité (légitime) ${ }^{19}$ ». C'était évidemment beaucoup moins enthousiaste que ce qui avait été écrit par les autres évêques. Les libéraux extrémistes exploitèrent ce qui leur sembla une divergence de vues entre Mgr Bourget et ses collègues du Québec; ils considérèrent même le premier comme opposé à la Confédération.

A partir de ce fait, Mgr Larocque, tout en se montrant compréhensif du mode d'agir de l'évêque de Montréal, l'invita cependant à prendre position d'une façon plus nette.

Il n'en est cependant pas moins vrai que nos ennemis communs continuent à faire du mal, en argumentant de cette prétendue division ou différence d'opinion, pour arriver à prouver que le droit est pour eux et que les évêques qui ont condamné plus ou moins directement leurs faits et gestes politiques, ont eu tort de le faire, et sont condamnés par le silence que vous avez gardé, prétendent-ils, relativement aux sujets abordés par les Mandements ou Lettres pastorales.

C'est pourquoi l'évêque de Saint-Hyacinthe termina sa lettre en priant Mgr Bourget de se dire publiquement en accord avec ses confrères dans l'épiscopat. Il affirma agir de cette façon a en conformité à l'opinion de quelques autres évêques et de plusieurs prêtres des mieux pensants et des plus distingués ${ }^{20}$ ».

La franchise et le courage dont venait de faire preuve Mgr Larocque vis-à-vis du vénérable $\mathrm{Mgr}$ Bourget ne portèrent pas les fruits attendus. Celui-ci répondit que ses deux lettres adressées au clergé et aux fidèles avaient paru produire leur effet, c'est-à-dire que

...tous ont compris que c'était pour eux un devoir de conscience de se soumettre à la confédération et qu'il leur fallait voter en conséquence... C'est donc à tort que l'on cherche à faire croire que je ne suis pas pour la confédération, sous prétexte que je me suis exprimé autrement qu'eux dans les documents précités 21.

18 Jean Hamelin, John Huot, Marcel Hamelin, op. cit., 49.

M.E.M. V, 25 juillet 1867, 236-244.

20 A.C.A.M., 295.103, 12 oct. 1867.

21 A.E.S.H., II, B. 2, 1867, 15 oct. 1867. 
Monseigneur Bourget évitait en fait de répondre au souhait de l'évêque de Saint-Hyacinthe. Celui-ci s'excusa de s'être peut-être mal exprimé. Il ne doutait pas que l'évêque de Montréal fût favorable à la Confédération; il avait seulement désiré qu'il se prononçât publiquement " sur la prétendue division" qui faisait apparaître les évêques en contradiction et ruiner l'autorité de leur parole.

Ma suggestion n'entre point dans vos idées; je respecte votre détermination, en vous priant de me pardonner de ne pouvoir changer d'opinion !! ! et mon opinion est que lo divicion qu'on nous impute fait un mal incalculable 22 .

De cette façon, on comprend mieux que le mois suivant, en novembre 1867 , après une réunion de plusieurs évêques canadiens à Toronto à l'occasion du sacre du nouvel évêque de Sandwich, où il fut question d'intervenir contre les journaux à tendance libérale, Mgr Larocque ait fait part à Mgr Laflèche de son désir "qu'un évêque parlât le premier et que tous les autres, chacun à sa manière, corroborassent ce qu'aurait dit celui-là ». Mais il ne voulait pas que le premier à parler fût Mgr Bourget et cela pour diverses raisons, dont la principale était qu'il serait alors obligé de revenir sur la question de la Confédération, au sujet de laquelle il avait paru d'accord avec les libéraux. Et Mgr Larocque d'ajouter:

Il n'est pas douteux pour personne que le quasi-silence de l'évêque de Montréal sur le grand événement que les quatre autres évêques du Bas-Canada ont si franchement abordé en comptant, je crois, sur son concours, a été la cause de l'impudence extrême avec laquelle les mauvais journaux ont attaqué les Mandements. Un homme important de Montréal s'est empressé de déclarer que si les évêques avaient été unanimes, le parti (aurait été) abattu et mort. Mais que l'attitude prise par l'évêque de Montréal l'avait sauvé23!

\section{ConcLusion}

En somme, les deux tentatives de Mgr Larocque d'amener Mgr Bourget à faire bloc avec le reste de l'épiscopat québécois, soit au début de juin alors qu'aucune lettre pastorale n'avait encore paru, soit au mois d'octobre à la suite de représailles verbales de la part de certaines gens à tendance libérale, n'eurent pas d'effets tangibles. L'évêque de Saint-Hyacinthe se faisait alors le porte-parole de Mgr Laflèche. C'était cependant avec conviction personnelle qu'il désirait un front uni de la part de l'épiscopat. Son optimisme vis-à-vis de la Confédération et son intrusion aussi avancée dans cette question d'ordre politique ne pouvaient pas être admis comme tels par l'évêque de Montréal. La différence dans le genre d'intervention est flagrante ${ }^{24}$, ce qui manifeste

22 A.E.S.H., $R L$, Série I, 5, 262-263, 16 oct. 1867.

23 Ibid., 289-294, 20 nov. 1867.

24 Walter Ullmann, The Quebec Bishops and Confederation, dans The Canadian Historical Review, XLIV, 3 (Sept. 1963) 232. 
surtout une différence d'audace vis-à-vis des ultralibéraux et une différence de contexte dans lequel chacun des évêques pouvait s'exprimer.

Lucien LemieuX, ptre.

Professeur d'Histoire de l'Eglise, Fac. de Théologie, Montréal.

\section{Abréviations}

ACAM Archives de la Chancellerie de l'archevêché de Montréal. AESH Archives de l'évêché de Saint-Hyacinthe.

MEM Mandements, lettres pastorales, circulaires et autres documents publiés dans le diocèse de Montréal depuis son érection jusqu'à l'année 1869.

MESH Mandements, lettres pastorales, circulaires des évêques de Saint-Hyacinthe.

RL Registres des lettres. 